\title{
Fratura do complexo zigomaticomaxilar por agressão física: relato de caso
}

\author{
Fracture of the zygomatic-maxillary complex by physical aggression: \\ case report
}

\section{Fractura del complejo zigomático-maxilar por agresión física: relato de caso}

\author{
Luara Teixeira COLOMBO1 \\ Gabriel Mulinari dos SANTOS ${ }^{1}$ \\ Paulo Zupelari GONÇALVES ${ }^{2}$ \\ André Luis da Silva FABRIS ${ }^{3}$ \\ Francisley Ávila SOUZA ${ }^{3}$ \\ Leonardo Perez FAVERANI \\ Idelmo Rangel GARCIA JÚNIOR ${ }^{3}$
}

\author{
${ }^{1}$ Mestrando em Cirurgia e Traumatologia Bucomaxilofacial, Faculdade de Odontologia de Araçatuba, \\ UNESP-Univ. Estadual Paulista, Araçatuba-SP, Brasil \\ ${ }^{2}$ Doutorando em Cirurgia e Traumatologia Bucomaxilofacial, Faculdade de Odontologia de Araçatuba, \\ UNESP-Univ. Estadual Paulista, Araçatuba-SP, Brasil \\ ${ }^{3}$ Professor em Cirurgia e Traumatologia Bucomaxilofacial, Faculdade de Odontologia de Araçatuba, \\ UNESP-Univ. Estadual Paulista, Araçatuba-SP, Brasil
}

\begin{abstract}
Resumo
O complexo zigomaticomaxilar ocupa a terceira posição dentre as fraturas faciais, acometendo principalmente jovens do sexo masculino e são decorrentes principalmente de acidente de trânsito e agressão física. A meta das cirurgias em face é minimizar o comprometimento estético, por isso utilizar acessos intrabucais e acessos cutâneos respeitando as linhas de Langer ou utilizar os ferimentos apresentados pelo paciente são condutas que devem ser empregadas. Este relato tem como objetivo apresentar o caso e discutir aspectos relativos à técnica cirúrgica que zelem tanto pelo aspecto funcional quanto pelo aspecto estético do paciente, mostrando uma opção para o tratamento de fraturas do complexo zigomático-maxilar.

Descritores: Traumatologia; Zigoma; Fixação de Fratura.
\end{abstract}

\begin{abstract}
The zygomatic-maxillary complex occupies the third position among the facial fractures, affecting mainly young men and are mainly due to traffic accident and physical aggression. The goal of surgeries in the face is to minimize aesthetic compromise, so using intra-buccal accesses and cutaneous accesses respecting Langer's lines or using the injuries presented by the patient are behaviors that should be employed. This report aims to present the case and discuss aspects related to the surgical technique that focus on both the functional aspect and the aesthetic aspect of the patient, showing an option for the treatment of fractures of the zygomatic-maxillary complex.
\end{abstract}

Descriptors: Traumatology; Zygoma; Fracture Fixation.

\begin{abstract}
Resumen
El complejo zigomático-maxilar ocupa la tercera posición entre las fracturas faciales, afectando principalmente a jóvenes del sexo masculino y son consecuencia principalmente de accidente de tránsito y agresión física. La meta de las cirugías en cara es minimizar el compromiso estético, por lo que utilizar accesos intra-bucales y accesos cutáneos respetando las líneas de Langer o utilizar las lesiones presentadas por el paciente son conductas que deben ser empleadas. Este relato tiene como objetivo presentar el caso y discutir aspectos relativos a la técnica quirúrgica que celem tanto por el aspecto funcional y el aspecto estético del paciente, mostrando una opción para el tratamiento de fracturas del complejo zigomático-maxilar.

Descriptores: Traumatología; Cigoma; Fijación de Fractura.
\end{abstract}

\section{INTRODUÇÃO}

A posição ocupada pelos ossos da face e o seu contorno irão influenciar na susceptibilidade dos mesmos a lesões. Devido a isso, complexo zigomático maxilar é a segunda região da face mais acometida por injúrias, sendo superada apenas pelos ossos nasais ${ }^{1,2}$. Fraturas nesse complexo podem levar a significantes alterações estéticas e funcionais, pois o seu posicionamento apresenta papel importante no contorno facial, além do posicionamento do globo ocular que é dependente, dentre outros fatores, do contorno da proeminência do zigoma ${ }^{3}$.

O complexo zigomático maxilar (CZM) é constituído pelo zigoma, também chamado de osso malar ou zigomático, que se apresenta como um osso piramidal e robusto. Além de quatro processos (temporal, orbital, maxilar e frontal) e de uma extensão óssea, o arco zigomático, formado por um prolongamento do zigoma (processo temporal do osso zigomático) e por outro do osso temporal (processo zigomático do osso temporal $)^{2,4}$.
Quando se trata de diagnóstico a atenção aos sinais e sintomas é fundamental, o Cirurgião Bucomaxilofacial (CBMF) deve procurar obter a história do trauma, realizar o exame físico e avaliar exames de imagem. Os sinais e sintomas predominantemente observados são: dormência no território de inervação do nervo infraorbitário, epistaxe, assimetria facial por afundamento da região zigomática, equimose subconjuntival, edema e hematoma palpebral, degrau em região infraorbitária, edema e equimose em mucosa jugal, degrau em pilar zigomático e diplopia ${ }^{5}$.

No que concerne aos exames de imagem para esse tipo de fratura, a radiografia em posição póstero-anterior de Waters e axial de Hirtz são as mais solicitadas, dado que se podem notar os contornos das órbitas, seios maxilar e frontal assim como os pilares zigomáticos. Outra característica que pode ser investigada é um possível velamento nos seios maxilares, muito comuns neste tipo de fratura ${ }^{2,6}$.

Fundamentados na experiência em 148 casos de 
fratura de zigoma, Manganello-Souza et al. $^{7}$ em 2003, classificaram as fraturas dessa região em três diferentes tipos, considerando tanto as alterações radiográficas quanto as funcionais. Sendo os três tipos:

Tipo I: pequeno deslocamento do osso (menor que $5 \mathrm{~mm}$ ); - fratura não cominutiva; ausência de disfunção ocular; tempo decorrido após o trauma de até 20 dias; tratamento conservador, com redução fechada ou uma placa em pilar zigomático.

Tipo II: grande deslocamento do osso (maior que $5 \mathrm{~mm}$ ); fratura cominutiva sem necessidade de reconstrução; presença de disfunção ocular; tempo decorrido após o trauma até 20 dias; tratamento prioriza redução aberta com um ou dois acessos cirúrgicos e fixação interna rígida em dois pontos.

Tipo III: grande deslocamento do osso (maior que $5 \mathrm{~mm}$ ); fratura cominutiva com necessidade de reconstrução; presença de disfunção ocular; fratura no corpo do zigoma; necessidade de fixar o arco zigomático; tempo decorrido após o trauma inferior a 20 dias; tratamento prioriza redução aberta com um, dois ou três acessos cirúrgicos e fixação interna rígida em três ou quatro pontos.

Apesar desta classificação, a opção em abordar uma fratura do complexo zigomático-maxilar com redução fechada ou aberta ainda é controverso. Alguns fatores como grau de deslocamento, a presença ou não de cominução, alterações funcionais e o tempo decorrido após a fratura devem ser levados em conta para eleger o tipo de tratamento ${ }^{3,8}$.

Assim, o objetivo desse trabalho é relatar um caso de fratura do complexo zigomático maxilar com rotação para medial, envolvimento do pilar zigomático, com tratamento por acessos no ferimento cortocontuso já presente e em região de sutura frontozigomática associado com acesso intrabucal.

\section{CASO CLÍNICO}

Paciente, gênero masculino, 19 anos, atendido na Santa Casa de Misericórdia de Araçatuba após ser vítima de agressão física, negando comorbidades, alergias e uso de medicamentos, apresentando ao exame físico: hiposfagma, edema e equimose periorbitária esquerda, perda de projeção malar, ferimentos corto-contuso em região zigomática à esquerda e superciliar ipsilateral, ferimento dermo-abrasivo em região de ângulo da mandíbula, não observando alteração nos movimentos oculares ou qualquer limitação de abertura bucal (Figura 1).

Ao exame de imagem, por intermédio da tomografia computadorizada em cortes coronal, axial e reconstrução pode-se constatar traço sugestivo de fratura na região de CZM esquerdo com rotação medial do fragmento, levando também a um envolvimento do pilar zigomático (Figura 2).

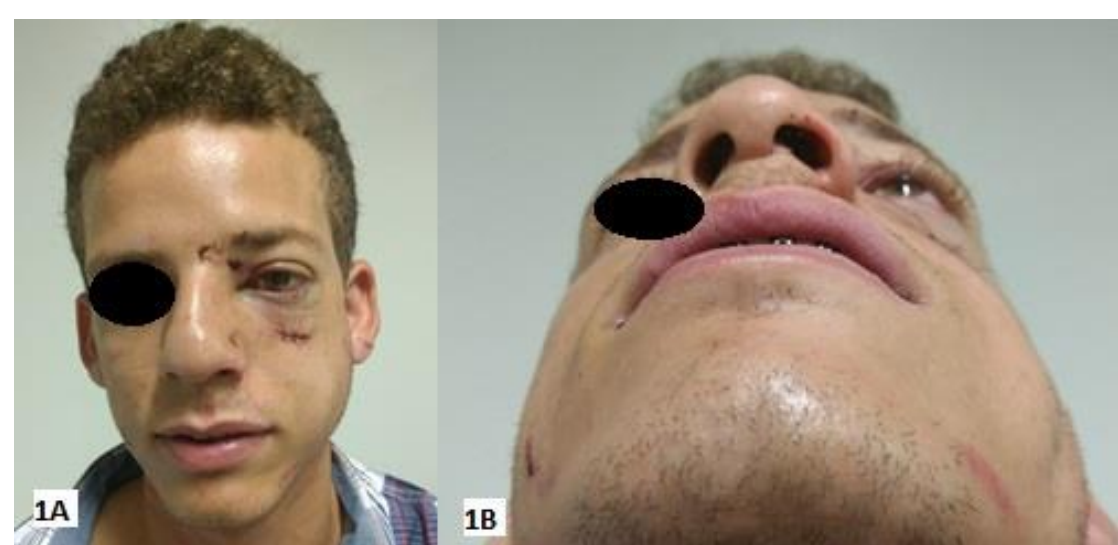

Figura 1: Pré-operatório. Evidenciando equimose periorbitária perda da projeção do complexo zigomaticomaxilar esquerdo em vista frontal (1 A) e vista ínfero-superior (1 B).

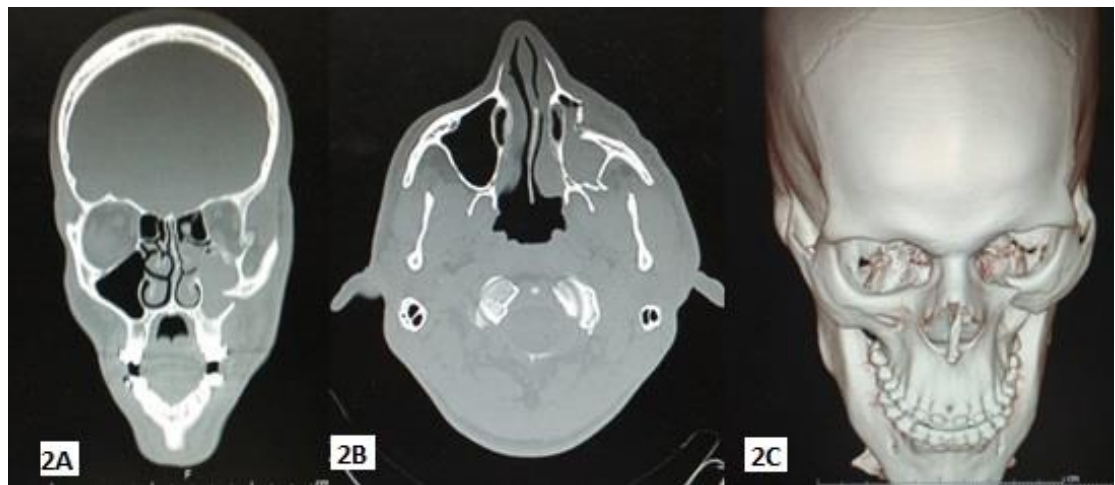

Figura 2: Tomografia computadorizada pré-operatória em cortes coronal (2 A), axial (2 B) e reconstrução tridimensional (2 C).

Após o exame clínico e imaginológico estabelecendo a fratura do CZM à esquerda com rotação medial do fragmento, compreendendo corpo do zigoma, sutura frontozigomática e pilar zigomático.

O procedimento cirúrgico foi realizado sob anestesia geral, entubação nasotraqueal. Foi realizado acesso vestibular intrabucal para posicionamento do parafuso de Bird para auxiliar na redução das fraturas, e também para exposição da fratura em pilar zigomático. $\mathrm{O}$ acesso transcutâneo supraorbital foi utilizado para expor a sutura fronto-zigomática. Por fim, foi realizado um acesso na região do corpo do zigoma através do ferimento cortocontuso que o paciente já possuía na região, expondo a fratura de corpo (Figura 3)

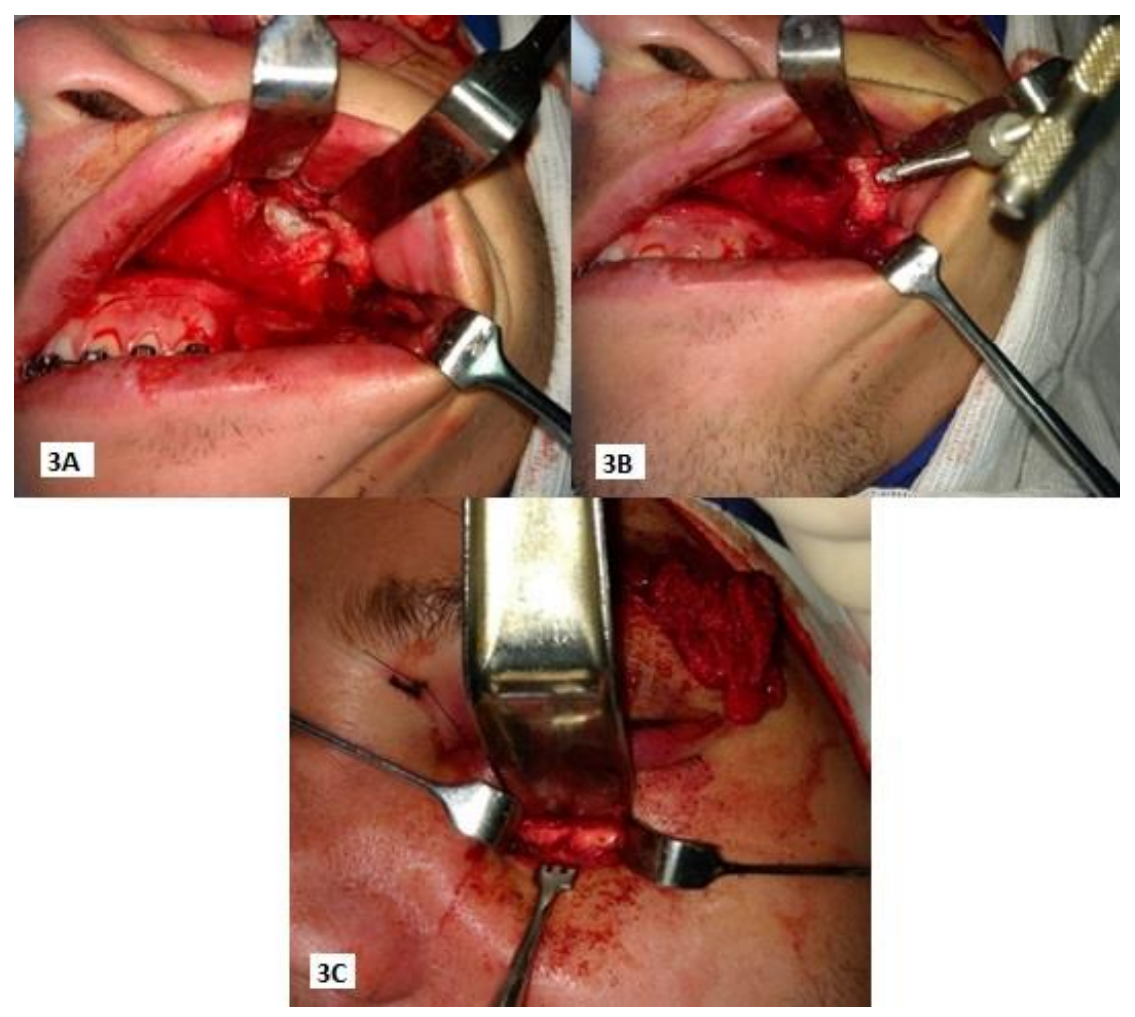

Figura 3: Transoperatório. Acesso vestibular (3 A), parafuso de Bird posicionado ( $3 \mathrm{~B}$ ) e acesso via ferimento corto-contuso (3 C).

Após realização do reposicionamento com parafuso de bird e redução dos cotos fraturados, optou-se pela fixação interna rígida. Para o pilar zigomático foi utilizada placa em "L" de 4 furos com intermediário e parafusos do sistema 2.0, na sutura fronto-zigomática foi empregada placa de 4 furos com intermediário e parafusos do sistema 1.5 , e na região de corpo do zigomático foi utilizada placa orbitária de 6 furos com intermediário e parafusos do sistema 1.5 (Figura 4).

No pós-operatório de 1 dia observou-se edema compatível com o procedimento cirúrgico, suturas e curativos em posição e ausência de sinais de sintomas de infecção local, e também relato de oclusão estável, ausência de diplopias pelo paciente. Radiograficamente, placas e parafusos com alinhamento dos segmentos fraturados (Figura 5). 


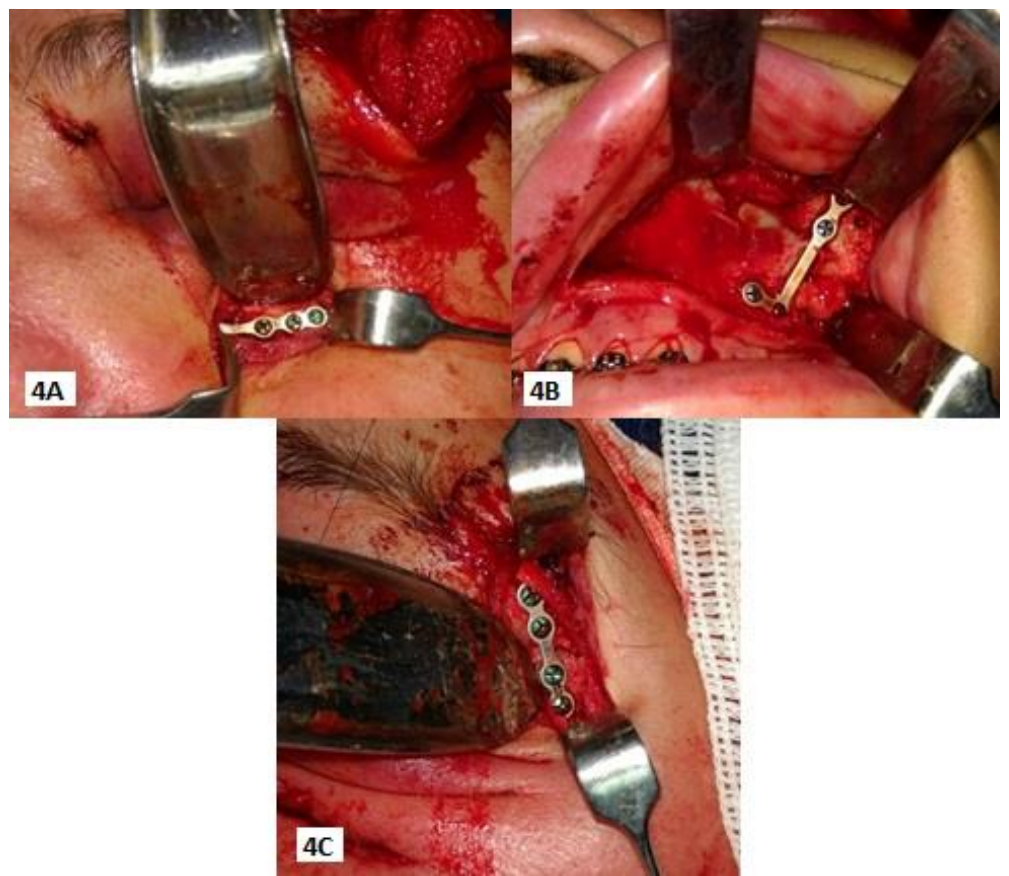

Figura 4: Fixação com placa orbitária de 6 furos com intermediário (sistema 1.5) (4 A), fixação com placa em "L" de 4 furos com intermediário (sistema 2.0) (4 B) e fixação com placa de 4 furos com intermediário (sistema 1.5) (4C).

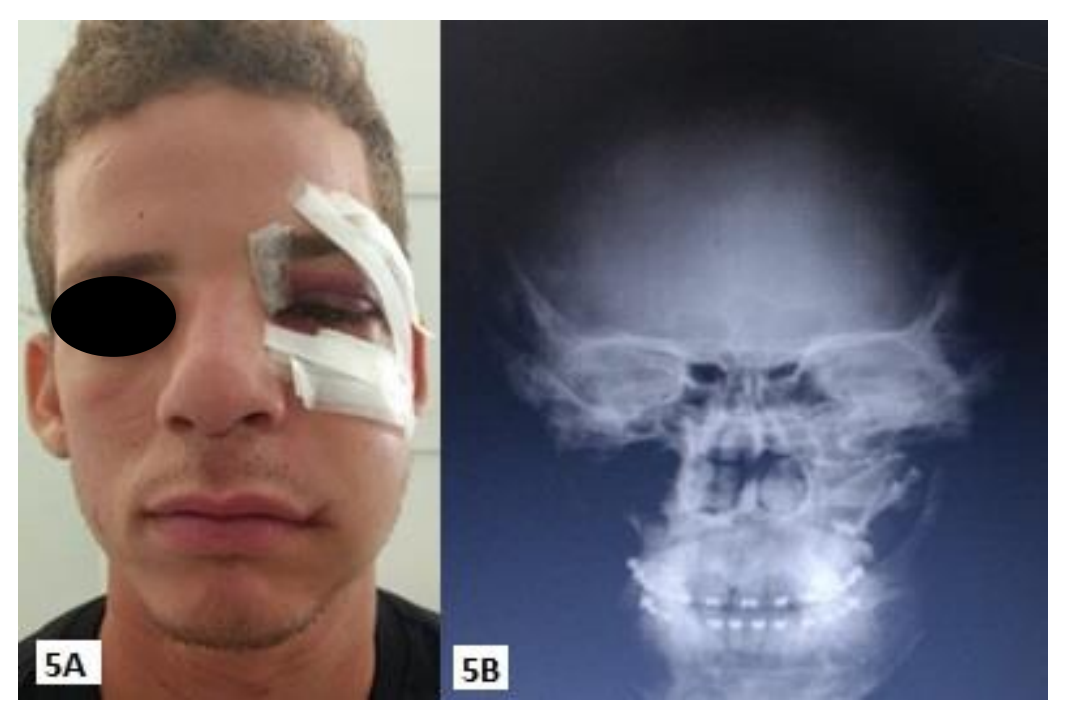

Figura 5: Aspectos físicos (5 A) e radiográficos (5 B) do pósoperatório de 1 dia

\section{DISCUSSÃO}

O CZM ocupa a terceira posição dentre as fraturas faciais, sendo que os indivíduos mais acometidos se encontram na faixa etária de 21 a $30 \operatorname{anos}^{9}$ e são do sexo masculino ${ }^{10}$. Com relação à etiologia dos traumas, os acidentes motociclísticos apresentaram a maior frequência, seguidos de agressão física e acidente ciclístico ${ }^{9}$. Sendo que no presente caso, o paciente apresentava características pessoais, semelhantes com as encontradas na literatura em fraturas CZM, de um paciente jovem, do sexo masculino e vítima de agressão física.

Apesar do tratamento desse tipo de fratura ser considerado controverso $^{3,8}$, o tratamento aberto com fixação interna rígida (FIR) continua a ser o principal para abordagem das fraturas de $\mathrm{CZM}^{11}$. Segundo diversos estudos $^{3,12,13}$ o acesso intra-bucal é satisfatório quando considerado a visibilidade, acessibilidade e facilidade na manipulação dos segmentos fraturados, além de não levar a formação de cicatrizes em área estética ${ }^{14}$. Corroborando com o caso exposto, onde se realizou o tratamento aberto com FIR e acesso intrabucal possibilitando melhor redução das fraturas.

Outro acesso muito legitimado pela literatura para a abordagem de fraturas desse complexo é o acesso infraorbiorbitário $^{3,15}$, que apresenta como desvantagem a formação de cicatriz que pela sua localização leva a comprometimento da harmonia facial. Entretanto, no presente estudo, foi utilizado o ferimento corto-contuso já apresentado pelo paciente, não levando a maiores prejuízos.

$\mathrm{O}$ uso do parafuso de Bird pelo acesso intra-bucal permitiu uma maior facilidade para a redução e manipulação do CZM sem necessitar de uma incisão adicional em pele, sendo positivo do ponto de vista estético e funcional.

\section{CONCLUSÃO}

Diante disso, do uso do ferimento corto-contuso para acesso a fratura e do uso do parafuso de Bird para redução da mesma, obteve-se um satisfatório resultado do ponto de vista estético e funcional, sendo possível restabelecer a projeção facial e oclusão do paciente.

\section{REFERÊNCIAS}

1. Tadj A, Kimble FW. Fractured zygomas. ANZ J Surg. 2003; 73(1-2)49-54.

2. Torres CS, Almeida DVF, Ribeiro Neto N, Oliveira MAM, Macedo Sobrinho JB. Rev Cir Traumatol BocoMaxilo-fac. 2008; 8(2):29-34.

3. Ellis E $3^{\text {rd }}$, Kittidumkerng W. Analysis of treatment for isolated zygomaticomaxillary complex fractures. J Oral Maxillofac Surg. 1996; 54(4):386-400.

4. Sassi LM, Dissenha JL, Bezeruska C, Guebur MI, Hepp V, Radaelli RL et al. Fraturas de zigomático: revisão de 50 casos. Rev Bras Cir Cabeça Pescoço. 2009; 38(4):246-7.

5. Paulesini Junior W, Farias LP, Aquati M, Rapoporat A, Leporace AA. Fratura de complexo zigomático: relato de caso. Rev Odontol UNICID. 2008; 20(3): 301-6.

6. Oliveira JAGP. Fratura do arco zigomático: relato de aso utilizando o acesso pré-auricular. Rev Cir Traumatol Buco-Maxilo-fac. 2009;9(1):47-52.

7. Manganello-Souza LC, Silva AAF, Pacheco DFS. Zygomatic and orbitozygomatic fractures. Rev Bras Cir Plást. 2003; 18(2):24-30.

8. Manson PN, Markowitz B, Mirvis S, Dunham M, Yaremchuk M. Toward CT-based facial fracture treatment. Plast Reconstr Surg. 1990; 85(2):202-12.

9. Mendes BC, Benetti IM, Aranega AM, Brandini DA, Souza FA, Bassi AP. Estudo epidemiológico das fraturas de CZM no período de 2006 a 2011 na região de Araçatuba-SP. Arch Health Invest. 2013; 2 (Esp 2):202.

10. Obuekwe O, Owotade F, Osayuwu O. Etiology and pattern of zygomatic complex fractures: a retrospective study. J Natl Med Assoc. 2005; 97(7):992-6.

11. Cortese A, Caggiano M, Carlino F, Pantaleo G. Zygomatic fractures: Technical modifications for better aesthetic and functional results in older patients. Int $\mathbf{J}$ Surg; 33(Suppl 1):S9-15.

12. Calderoni DR, Guidi MC, Kharmandaiyan P, Nunes PHF. Seven-year institutional experience in the surgical treatment of orbito-zygomatic fractures. J Craniomaxillofac Surg. 2011; 39(8):593-9.

13. Adam AADM, Zhi L, Bing LZ, Xing WUZ. Evaluation of treatment of zygomatic bone and zygomatic arch fractures: a retrospective study of 10 years. J Maxillofac Surg. 2012; 11(2):171-6.

14. Schnetler JFC. A technique for reducing fractures of the zygomatic complex under local anaesthesia. Br J Oral Maxillofac Surg. 1990; 28(3):168-71.

15. Habal MB. The orbits: it is less important what you put in than how you secure it. J Craniofac Surg. 2010; 21(4):965-6. 


\section{CONFLITO DE INTERESSES}

Os autores declaram não haver conflitos de interesse.

\section{AUTOR PARA CORRESPONDÊNCIA}

Gabriel Mulinari dos Santos

gabriel_mulinari@hotmail.com

Submetido em 12/05/2017

Aceito em 08/07/2017 